\title{
SNP interactions of Helicobacter pylori-related host genes PGC, PTPN11, IL1B, and TLR4 in susceptibility to gastric carcinogenesis
}

\author{
Caiyun He ${ }^{1,2}$, Huakang $\mathrm{Tu}^{3}$, Liping Sun ${ }^{1}$, Qian $\mathrm{Xu}^{1}{ }^{1}$, Yuehua Gong ${ }^{1}$, Jingjing Jing ${ }^{1}$, \\ Nannan Dong ${ }^{1}$ and Yuan Yuan ${ }^{1}$ \\ ${ }^{1}$ Tumor Etiology and Screening Department of Cancer Institute and General Surgery, the First Affiliated Hospital of China \\ Medical University, and Key Laboratory of Cancer Etiology and Prevention (China Medical University), Liaoning Provincial \\ Education Department, Shenyang, China \\ 2 Department of Molecular Diagnostics, Sun Yat-Sen University Cancer Center, State Key Laboratory of Oncology in South \\ China, Collaborative Innovation Center for Cancer Medicine, Guangzhou, China \\ ${ }^{3}$ Department of Epidemiology, Rollins School of Public Health, Emory University, Atlanta, GA, USA \\ Correspondence to: Yuan Yuan, email: yyuan@mail.cmu.edu.cn \\ Keywords: Helicobacter pylori, gastric cancer, atrophic gastritis, susceptibility, interaction \\ Received: March 24, $2015 \quad$ Accepted: May 13, $2015 \quad$ Published: May 22, 2015
}

This is an open-access article distributed under the terms of the Creative Commons Attribution License, which permits unrestricted use, distribution, and reproduction in any medium, provided the original author and source are credited.

\section{ABSTRACT}

A series of host genes that respond to Helicobacter pylori (H. pylori) infection are involved in the process of gastric carcinogenesis. This study sought to examine interactions among polymorphisms of $H$. pylori-related genes PGC, PTPN11, TLR4, and $I L 1 B$ and assess whether their interaction effects were modified by $H$. pylori infection. Thirteen polymorphisms of the aforementioned genes were genotyped by the Sequenom MassARRAY platform in 714 gastric cancer patients, 907 atrophic gastritis cases and 1276 healthy control subjects. When we considered the host genetic effects alone, gene-gene interactions consistently decreased the risks of gastric cancer and/or atrophic gastritis, including three two-way interactions: PGC rs6912200-PTPN11 rs12229892, PGC rs4711690-IL1B rs1143623 and PTPN11 rs12229892-IL1B rs1143623 and a three-way interaction: PGC rs4711690-PGC rs6912200-PTPN11 rs12229892. When the effect modification of $H$. pylori infection was evaluated, the cumulative effects of the aforementioned three-way interaction on atrophic gastritis susceptibility switched from being beneficial to being risky by the status of $\boldsymbol{H}$. pylori infection. These data showed that SNP interactions among $\boldsymbol{H}$. pylorirelated genes PGC, PTPN11, and IL1B, are associated with susceptibility to gastric carcinogenesis. Moreover, we provided important hints of an effect modification by $H$. pylori infection on the cumulative effect of PGC and PTPN11 polymorphisms. Functional experiments and further independent large-scale studies especially in other ethnic populations are still needed to confirm our results.

\section{INTRODUCTION}

The genetic basis of susceptibility to gastric cancer is the cumulative result of germ-line variations at many different loci, with each gene only having a small effect [1]. In typical case-control association studies of gastric cancer, candidate genes are examined either by evaluating one marker at a time or by forming haplotypes over multiple neighboring loci in and around one gene [2]. There are limited data regarding the influence of gene- gene interactions on gastric cancer risk. One important type of gene-gene interaction is epistasis, in which the genes interact with one another and modify each other's behavior [3]. In fact, understanding epistatic interactions may be the key to understanding complex diseases. Knowledge of gene-gene interactions could reveal substantial hidden heritability within the architecture of gastric cancer susceptibility [4].

Helicobacter pylori is a confirmed environmental risk factor for gastric cancer and its precursors, such 
as atrophic gastritis and dysplasia [5]. It exhibits carcinogenic effects on gastric epithelial cells and these effects are mediated by a series of virulence factors and toxic components [6]. The host response to these bacterial components differs between individuals and is extremely important for determining gastric cancer predisposition [6]. It is becoming increasingly clear that several specific host genes, such as $P G C$ (pepsinogen C), PTPN11 (protein tyrosine phosphatase, non-receptor type 11), TLR4 (Tolllike receptor 4), and $I L 1 B$ (interleukin-1B), are involved in the response to $H$. pylori infection and are currently identified as susceptibility genes for gastric cancer $[6,7]$.

PTPN11 and TLR4 are crucial components of the gastric epithelial cell signaling pathway and respond to the virulence factors LPS (lipopolysaccharide) and CagA (cytotoxin-associated antigen) of $H$. pylori, respectively[8]. Such host-microbe interactions can activate the NF-kB (nuclear factor-kappa B) and MAPK (mitogen-activated protein kinase) signaling pathways, which promote the production of the proinflammatory factor IL-1 $\beta$ or induce aberrant apoptosis or proliferation[8-11]. PGC, a well-known biomarker for the differentiation of gastric epithelium cells, has recently been recognized as a surrogate for H. pylori infection in the stomach [12-14]. The aforementioned host genes appear to have pleiotropic effects on the signal transduction of inflammatory or immune reactions, proliferation, apoptosis, and cell differentiation [8, 14-16].

Individual genetic effects of 13 single nucleotide polymorphisms (SNPs) in PGC, PTPN11, TLR4, and IL1B on the susceptibility to gastric cancer and atrophic gastritis have been reported in our previous studies [7, 17]. In this study, we investigated the potential gene-gene interactions among those SNPs and assessed whether the effects of these interactions were modified by $H$. pylori infection. To our knowledge, this is the first study to investigate interactions between $H$. pylori-related genes as risk factors for gastric carcinogenesis.

\section{RESULTS}

\section{Main effects of single polymorphisms of PGC, PTPN11, TLR4, and IL1B}

In our previous studies [7, 17], we found that the genotype frequencies of five tagSNPs of $P G C$ (rs4711690, rs6458238, rs9471643, rs3789210, and rs6939861) in gastric cancer and/or atrophic gastritis were significantly different from those in controls. Moreover, $H$. pylori infection status affected the ORs of three tagSNPs (PGC rs4711690, $P G C$ rs6912200, and PTPN11 rs12229892) for the development of gastric cancer or atrophic gastritis. There was no overall genetic effect on risk for $P G C$ rs6912200 and rs6941539; PTPN11 rs12229892;
IL1B rs1143623, rs1143627 and rs1143643; or TLR4 rs11536878 and rs10983755 in our study population.

\section{Two-way interactions between polymorphisms of $P G C, P T P N 11, T L R 4$, and IL1B}

Using a combined genotype comprising the most common SNP for each gene, two-way gene-gene interactions among the 13 tagSNPs of our genes of interest were assessed. In the two-way interaction analyses involving $P G C$ (Table 1 and Supplementary Tables 2 and 3 ), the most significant interaction was between $P G C$ rs6912200 and PTPN11 rs12229892. This interaction was associated with altered risks for the development of both gastric cancer and atrophic gastritis (gastric cancer risk: $P$ value for interaction $=0.017$, interaction index $=1.96$; atrophic gastritis risk: $P$ value for interaction $=0.010$, interaction index $=1.97$ ). In addition, $P G C$ rs4711690 showed a significant interaction with $I L 1 B$ rs1143623 in relation to gastric cancer risk $(P$ value for interaction $=$ 0.047 , interaction index $=0.65)$. In the two-way analyses involving PTPN11 (Table 2), PTPN11 rs12229892 showed a significant interaction with $I L 1 B$ rs1143623, influencing gastric cancer risk ( $P$ value for interaction $=0.034$, interaction index $=1.64)$. In the two-way analyses between $T L R 4$ and $I L-1 B$, no statistically significant interaction was observed (Supplementary Table 4).

\section{Epistatic effects of two-way interactions}

Among the four polymorphisms involved in significant pairwise interactions, $P G C$ rs6912200, PTPN11 rs12229892 and $I L 1 B$ rs1143623 had no overall main effect on disease risk $[7,17]$. We therefore examined the epistatic effects between pairs of interacting factors (Table 3). For PGC rs6912200 and PTPN11 rs12229892, TC/TT genotypes at rs6912200 and GA/AA genotypes at rs12229892 each conferred a reduced risk of gastric cancer and atrophic gastritis, but not if they were present together. For $P G C$ rs4711690 and $I L 1 B$ rs 1143623 , rs4711690 $\mathrm{GG} / \mathrm{GC}$ genotypes were associated with a reduced risk of gastric cancer and atrophic gastritis, but only in the presence of GC/CC genotypes at rs1143623. For PTPN11 rs12229892 and $I L 1 B$ rs1143623, rs1143623 GC/CC genotypes were associated with a reduced gastric cancer risk only in the absence of rs 12229892 GA/AA genotypes. These observations suggest that $P G C$ rs6912200, PTPN11 rs12229892, and $I L 1 B$ rs1143623 individually have no main effect but demonstrate pairwise epistatic interactions. 
Table 1: Two-way interaction effect between $P G C$ tagSNPs and $P T P N 11$ and $I L 1 B$ tagSNPs on the risks of gastric cancer and atrophic gastritis

\begin{tabular}{|c|c|c|c|c|c|c|c|c|c|}
\hline \multirow{3}{*}{$\begin{array}{l}P G C \\
\text { tagSNP }\end{array}$} & & \multicolumn{4}{|c|}{ For GA vs. CON } & \multicolumn{4}{|c|}{ For GC vs. CON } \\
\hline & & \multicolumn{2}{|c|}{ PTPN11 rs12229892 } & \multicolumn{2}{|c|}{$I L 1 B$ rs 1143623} & \multicolumn{2}{|c|}{ PTPN11 rs12229892 } & \multicolumn{2}{|l|}{$I L 1 B$ rs 1143623} \\
\hline & & GG & GA/AA & GG & GA/AA & GG & GA/AA & GG & GA/AA \\
\hline \multicolumn{10}{|l|}{ rs 4711690} \\
\hline \multirow[t]{2}{*}{$\mathrm{CC}$} & Controls/Cases & $227 / 129$ & $476 / 290$ & $252 / 143$ & $452 / 277$ & $227 / 180$ & $476 / 359$ & $252 / 183$ & $452 / 357$ \\
\hline & $\mathrm{OR}(95 \% \mathrm{Cl})$ & 1 (ref) & $1.21(0.90,1.61)$ & 1(ref) & $1.18(0.89,1.56)$ & 1(ref) & $0.89(0.69,1.15)$ & 1 (ref) & $1.05(0.81,1.35)$ \\
\hline \multirow[t]{3}{*}{$\mathrm{CG} / \mathrm{GG}$} & Controls/Cases & $164 / 93$ & $393 / 196$ & $198 / 117$ & $362 / 171$ & $164 / 103$ & $393 / 258$ & $198 / 129$ & $362 / 232$ \\
\hline & $\mathrm{OR}(95 \% \mathrm{Cl})$ & $1.08(0.75,1.57)$ & $0.88(0.65,1.20)$ & $1.08(0.77,1.52)$ & $0.82(0.61,1.12)$ & $0.70(0.50,0.98)$ & $0.73(0.56,0.96)$ & $0.80(0.59,1.10)$ & $0.80(0.61,1.05)$ \\
\hline & & \multicolumn{2}{|c|}{$\begin{array}{l}P \text { for interaction }=0.089 \text {, interaction } \\
\text { index }=0.68\end{array}$} & \multicolumn{2}{|c|}{$\begin{array}{l}P \text { for interaction }=0.047 \\
\text { interaction index }=0.65\end{array}$} & \multicolumn{2}{|c|}{$\begin{array}{l}P \text { for interaction }=0.420, \text { interaction } \\
\text { index }=1.18\end{array}$} & \multicolumn{2}{|c|}{$\begin{array}{l}P \text { for interaction }=0.797, \text { interaction } \\
\text { index }=0.95\end{array}$} \\
\hline \multicolumn{10}{|l|}{ rs6458238 } \\
\hline \multirow[t]{2}{*}{ GG } & Controls/Cases & $325 / 183$ & $710 / 402$ & $361 / 215$ & $678 / 369$ & $325 / 246$ & $710 / 526$ & $361 / 264$ & $678 / 509$ \\
\hline & $\mathrm{OR}(95 \% \mathrm{Cl})$ & 1 (ref) & $1.04(0.82,1.33)$ & 1(ref) & $0.93(0.74,1.18)$ & 1(ref) & $0.92(0.74,1.14)$ & $1(\mathrm{ref})$ & $0.98(0.80,1.21)$ \\
\hline \multirow[t]{3}{*}{ AG/AA } & Controls/Cases & $67 / 39$ & $161 / 87$ & $90 / 47$ & $138 / 79$ & $67 / 39$ & $161 / 92$ & $90 / 49$ & $138 / 83$ \\
\hline & $\mathrm{OR}(95 \% \mathrm{CI})$ & $0.96(0.60,1.55)$ & $0.95(0.67,1.35)$ & $0.80(0.52,1.22)$ & $0.96(0.67,1.37)$ & $0.65(0.41,1.04)$ & $0.70(0.51,0.97)$ & $0.64(0.42,0.96)$ & $0.78(0.56,1.10)$ \\
\hline & & \multicolumn{2}{|c|}{$\begin{array}{l}P \text { for interaction }=0.860 \text {, interaction } \\
\text { index }=0.95\end{array}$} & \multicolumn{2}{|c|}{$\begin{array}{l}P \text { for interaction }=0.355 \\
\text { interaction index }=1.29\end{array}$} & \multicolumn{2}{|c|}{$\begin{array}{l}P \text { for interaction }=0.573 \text {, interaction } \\
\text { index }=1.17\end{array}$} & \multicolumn{2}{|c|}{$\begin{array}{l}P \text { for interaction }=0.405 \text {, interaction } \\
\text { index }=1.25\end{array}$} \\
\hline \multicolumn{10}{|l|}{ rs 9471643} \\
\hline $\mathrm{GG} / \mathrm{CC}$ & Controls/Cases & $234 / 141$ & $536 / 316$ & $272 / 164$ & $498 / 293$ & $234 / 185$ & $536 / 385$ & $272 / 201$ & $498 / 367$ \\
\hline & $\mathrm{OR}(95 \% \mathrm{Cl})$ & 1 (ref) & $1.07(0.81,1.41)$ & 1 (ref) & $1.09(0.84,1.43)$ & 1 (ref) & $0.87(0.70,1.12)$ & 1 (ref) & $1.01(0.80,1.29)$ \\
\hline $\mathrm{GC}$ & Controls/Cases & $157 / 80$ & $332 / 173$ & $176 / 96$ & $315 / 155$ & $157 / 99$ & $332 / 231$ & $176 / 109$ & $315 / 223$ \\
\hline & $\mathrm{OR}(95 \% \mathrm{Cl})$ & $0.86(0.59,1.25)$ & $0.82(0.60,1.12)$ & $0.95(0.67,1.35)$ & $0.77(0.57,1.05)$ & $0.78(0.54,1.07)$ & $0.80(0.60,1.05)$ & $0.84(0.61,1.16)$ & $0.90(0.69,1.17)$ \\
\hline & & $\begin{array}{l}P \text { for interaction }= \\
\text { index }=0.90\end{array}$ & 0.639 , interaction & $\begin{array}{l}\mathrm{P} \text { for interaction } \\
\text { interaction inde }\end{array}$ & $\begin{array}{l}=0.183 \\
\mathrm{x}=0.74\end{array}$ & $\begin{array}{l}P \text { for interaction } \\
\text { index }=1.19\end{array}$ & $\mathrm{n}=0.395$, interaction & $\begin{array}{l}P \text { for interacti } \\
\text { index }=1.05\end{array}$ & on $=0.801$, interaction \\
\hline rs3789210 & & & & & & & & & \\
\hline $\mathrm{CC}$ & Controls/Cases & $199 / 162$ & $470 / 323$ & $241 / 183$ & $429 / 302$ & $199 / 201$ & $407 / 408$ & $241 / 203$ & $429 / 408$ \\
\hline & $\mathrm{OR}(95 \% \mathrm{CI})$ & $1(\mathrm{ref})$ & $0.88(0.67,1.15)$ & $1(\mathrm{ref})$ & $0.93(0.71,1.20)$ & 1(ref) & $0.77(0.60,0.99)$ & 1 (ref) & $1.06(0.93,1.36)$ \\
\hline $\mathrm{GC} / \mathrm{GG}$ & Controls/Cases & $76 / 44$ & $144 / 111$ & $79 / 50$ & $141 / 104$ & $76 / 47$ & $144 / 115$ & $79 / 67$ & $141 / 95$ \\
\hline & $\mathrm{OR}(95 \% \mathrm{CI})$ & $0.73(0.46,1.17)$ & $0.95(0.67,1.350$ & $0.77(0.50,1.19)$ & $0.99(0.71,1.40)$ & $0.58(0.38,0.91)$ & $0.69(0.49,0.96)$ & $0.92(0.62,1.36$ & $0.76(0.54,1.06)$ \\
\hline & & $\begin{array}{l}P \text { for interaction }= \\
\text { index }=1.48\end{array}$ & $=0.162$, interaction & $\begin{array}{l}\text { P for interaction } \\
\text { interaction inde }\end{array}$ & $\begin{array}{l}=0.230 \\
\mathrm{x}=1.39\end{array}$ & $\begin{array}{l}\mathrm{P} \text { for interaction } \\
\text { index }=1.54\end{array}$ & $\mathrm{n}=0.110$, interaction & $\begin{array}{l}P \text { for interacti } \\
\text { index }=0.77\end{array}$ & on $=0.317$, interaction \\
\hline rs6912200 & & & & & & & & & \\
\hline $\mathrm{CC}$ & Controls/Cases & $55 / 53$ & $172 / 113$ & $77 / 66$ & $148 / 98$ & $55 / 71$ & $172 / 127$ & $77 / 72$ & $148 / 126$ \\
\hline & $\mathrm{OR}(95 \% \mathrm{CI})$ & 1(ref) & $0.56(0.35,0.91)$ & 1 (ref) & $0.71(0.45,1.11)$ & 1 (ref) & $0.51(0.32,0.79)$ & 1 (ref) & $0.85(0.56,1.31)$ \\
\hline $\mathrm{CT} / \mathrm{TT}$ & Controls/Cases & $217 / 152$ & $442 / 319$ & $243 / 164$ & $419 / 308$ & $217 / 176$ & $442 / 393$ & $243 / 196$ & $419 / 374$ \\
\hline & $\mathrm{OR}(95 \% \mathrm{CI})$ & $0.57(0.36,0.91)$ & $0.60(0.41,0.98)$ & $0.68(0.45,1.03)$ & $0.79(0.53,1.16)$ & $0.66(0.43,1.01)$ & $0.66(0.44,0.98)$ & $0.90(0.60,1.33$ & $0.97(0.67,1.41)$ \\
\hline & & $\begin{array}{l}P \text { for interactior } \\
\text { index }=1.96\end{array}$ & $=0.017$, interaction & $\begin{array}{l}\mathrm{P} \text { for interaction } \\
\text { interaction inde }\end{array}$ & $\begin{array}{l}=0.063 \\
x=1.64\end{array}$ & $\begin{array}{l}P \text { for interactio } \\
\text { index }=1.97\end{array}$ & on $=0.010$, interactiol & $\begin{array}{l}P \text { for interacti } \\
\text { index }=1.27\end{array}$ & on $=0.341$, interaction \\
\hline rs6939861 & & & & & & & & & \\
\hline GG & Controls/Cases & $117 / 87$ & $272 / 159$ & $146 / 92$ & $242 / 153$ & $117 / 98$ & $272 / 197$ & $146 / 108$ & $242 / 190$ \\
\hline & $\mathrm{OR}(95 \% \mathrm{CI})$ & 1 (ref) & $0.76(0.53,1.10)$ & 1 (ref) & $0.98(0.69,1.40)$ & 1(ref) & $0.78(0.55,1.10)$ & 1(ref) & $1.00(0.72,1.40)$ \\
\hline $\mathrm{AG} / \mathrm{AA}$ & Controls/Cases & $136 / 112$ & $321 / 261$ & $163 / 131$ & $295 / 242$ & $136 / 142$ & $321 / 310$ & $163 / 155$ & $295 / 295$ \\
\hline & $\mathrm{OR}(95 \% \mathrm{CI})$ & $1.06(0.71,1.58)$ & $1.12(0.79,1.58)$ & $1.26(0.87,1.82)$ & $1.33(0.96,1.86)$ & $1.25(0.85,1.82)$ & $1.07(0.77,1.49)$ & $1.27(0.90,1.8$ & 1) $\quad 1.32(0.96,1.81)$ \\
\hline & & $\begin{array}{l}P \text { for interaction }= \\
\text { index }=1.39\end{array}$ & $=0.186$, interaction & $\begin{array}{l}\mathrm{P} \text { for interaction } \\
\text { interaction inde }\end{array}$ & $\begin{array}{l}=0.728 \\
x=1.09\end{array}$ & $\begin{array}{l}P \text { for interaction } \\
\text { index }=1.10\end{array}$ & $\mathrm{n}=0.687$, interaction & $\begin{array}{l}P \text { for interacti } \\
\text { index }=1.03\end{array}$ & on $=0.886$, interaction \\
\hline rs6941539 & & & & & & & & & \\
\hline $\mathrm{CC}$ & Controls/Cases & $203 / 143$ & $434 / 316$ & $236 / 166$ & $402 / 293$ & $203 / 169$ & $434 / 359$ & $236 / 190$ & $402 / 339$ \\
\hline $\mathrm{CT} / \mathrm{TT}$ & $\begin{array}{l}\text { OR }(95 \% \mathrm{CI}) \\
\text { Controls/Cases }\end{array}$ & $\begin{array}{l}1(\mathrm{ref}) \\
70 / 63\end{array}$ & $\begin{array}{l}1.04(0.78,1.37) \\
176 / 117\end{array}$ & $\begin{array}{l}1(\mathrm{ref}) \\
82 / 67\end{array}$ & $\begin{array}{l}1.08(0.82,1.41) \\
164 / 112\end{array}$ & $\begin{array}{l}1(\mathrm{ref}) \\
70 / 77\end{array}$ & $\begin{array}{l}0.89(0.69,1.16) \\
176 / 161\end{array}$ & $\begin{array}{l}1(\text { ref) } \\
82 / 79\end{array}$ & $\begin{array}{l}0.99(0.77,1.28) \\
164 / 159\end{array}$ \\
\hline & $\mathrm{OR}(95 \% \mathrm{Cl})$ & $1.28(0.83,1.97)$ & $0.98(0.70,1.38)$ & $1.23(0.82,1.86)$ & $1.03(0.74,1.44)$ & $1.30(0.87,1.96)$ & $0.98(0.72,1.34)$ & $1.14(0.77,1.67)$ & $1.17(0.86,1.48)$ \\
\hline & & $\begin{array}{l}P \text { for interaction }= \\
\text { index }=0.75\end{array}$ & $=0.275$, interaction & $\begin{array}{l}\text { P for interaction= } \\
\text { interaction index }\end{array}$ & $\begin{array}{l}=0.335 \\
=0.78\end{array}$ & $\begin{array}{l}P \text { for interaction }=0 \\
\text { index }=0.84\end{array}$ & 0.494 , interaction & $\begin{array}{l}\mathrm{P} \text { for interaction }= \\
\text { index }=1.04\end{array}$ & 0.882 , interaction \\
\hline
\end{tabular}

All tests were adjusted by age, sex and $H$. pylori infection. Statistically significant interactions were highlighted in bold (P values $<0.05)$. Abbreviation: $\mathrm{GC}$, gastric cancer; $\mathrm{GA}$, atrophic gastritis; $\mathrm{CON}$, healthy controls. 
Table 2: Two-way interaction effect between PTPN11 tagSNPs and $I L 1 B$ and TLR4 tagSNPs on risks of gastric cancer and atrophic gastritis

\begin{tabular}{|c|c|c|c|c|c|c|c|c|c|c|}
\hline \multirow{2}{*}{$\begin{array}{c}P G C \\
\text { tagSNP }\end{array}$} & \multicolumn{2}{|c|}{$I L 1 B \operatorname{rs} 1143623$} & \multicolumn{2}{|c|}{$I L 1 B \operatorname{rs} 1143627$} & \multicolumn{2}{|c|}{$I L 1 B \operatorname{rs} 1143643$} & \multicolumn{2}{|c|}{ TLR4 rs10983755 } & \multicolumn{2}{|c|}{ TLR4 rs1 1536878} \\
\hline & GG & $\mathrm{GC} / \mathrm{CC}$ & TT & $\mathrm{TC} / \mathrm{CC}$ & AA & $\mathrm{AG} / \mathrm{GG}$ & GG & $\mathrm{GA} / \mathrm{AA}$ & $\mathrm{CC}$ & $\mathrm{CA} / \mathrm{AA}$ \\
\hline \multicolumn{11}{|c|}{ For GC vs. CON } \\
\hline \multicolumn{11}{|c|}{ PTPN11 rs12229892 } \\
\hline \multirow[t]{2}{*}{ GG } & $128 / 87$ & $263 / 135$ & $101 / 66$ & $287 / 154$ & $111 / 62$ & $280 / 260$ & $167 / 115$ & $164 / 100$ & $315 / 175$ & $74 / 42$ \\
\hline & $1(\mathrm{ref})$ & $0.69(0.47,1.02)$ & 1 (ref) & $0.70(0.47,1.06)$ & $1(\mathrm{ref})$ & $0.97(0.64,1.45)$ & $1(\mathrm{ref})$ & $0.72(0.50,1.06)$ & $1(\mathrm{ref})$ & $0.97(0.61,1.55)$ \\
\hline \multirow[t]{3}{*}{$\mathrm{GA} / \mathrm{AA}$} & $321 / 173$ & $553 / 313$ & $239 / 129$ & $630 / 354$ & $241 / 132$ & $630 / 356$ & $364 / 240$ & $358 / 231$ & $693 / 383$ & $180 / 96$ \\
\hline & $0.74(0.51,1.07)$ & $0.84(0.60,1.18)$ & $0.72(0.47,1.10)$ & $0.81(0.56,1.18)$ & $0.94(0.62,1.43)$ & $1.02(0.71,1.49)$ & $0.93(0.68,1.28)$ & $0.82(0.59,1.12)$ & $1.03(0.80,1.31)$ & $0.97(0.69,1.36)$ \\
\hline & $\begin{array}{l}\text { P for interactio } \\
\text { interaction i }\end{array}$ & $\begin{array}{l}.034 \\
x=1.64\end{array}$ & $\begin{array}{l}\text { P for interaction } \\
\text { index }=1.59\end{array}$ & .066 , interaction & $\begin{array}{l}P \text { for interaction } \\
\text { index }=1.13\end{array}$ & .634 , interaction & $\begin{array}{l}\mathrm{P} \text { for interaction } \\
\text { index }=1.22\end{array}$ & .397 , interaction & $\begin{array}{l}P \text { for interaction } \\
\text { index }=0.97\end{array}$ & 917 , interaction \\
\hline \multicolumn{11}{|c|}{ For GA vs. CON } \\
\hline \multicolumn{11}{|c|}{ PTPN11 rs12229892 } \\
\hline \multirow[t]{2}{*}{ GG } & $128 / 103$ & $263 / 181$ & $101 / 84$ & $287 / 200$ & $111 / 83$ & $280 / 202$ & $167 / 144$ & $164 / 122$ & $315 / 220$ & $74 / 63$ \\
\hline & $1(\mathrm{ref})$ & $0.84(0.59,1.18)$ & $1(\mathrm{ref})$ & $0.76(0.53,1.10)$ & $1(\mathrm{ref})$ & $0.96(0.66,1.37)$ & 1 (ref) & $0.83(0.59,1.17)$ & $1(\mathrm{ref})$ & $1.29(0.86,1.93)$ \\
\hline \multirow[t]{3}{*}{ GA/AA } & $321 / 207$ & $553 / 411$ & $239 / 154$ & $630 / 460$ & $241 / 154$ & $630 / 464$ & $364 / 286$ & $358 / 290$ & $693 / 501$ & $180 / 116$ \\
\hline & $0.77(0.55,1.07)$ & $0.86(0.63,1.18)$ & $0.70(0.48,1.02)$ & $0.78(0.55,1.09)$ & $0.80(0.55,1.17)$ & $0.94(0.67,1.31)$ & $0.85(0.64,1.14)$ & $0.83(0.62,1.11)$ & $0.99(0.80,1.24)$ & $0.90(0.66,1.23)$ \\
\hline & $\begin{array}{l}P \text { for interaction } \\
\text { index }=1.34\end{array}$ & 165, interaction & $\begin{array}{l}\text { P for interaction } \\
\text { index }=1.47\end{array}$ & .090 , interaction & $\begin{array}{l}P \text { for interaction } \\
\text { index }=1.22\end{array}$ & 369 , interaction & $\begin{array}{l}P \text { for interaction } \\
\text { index }=1.19\end{array}$ & 424, interaction & $\begin{array}{l}P \text { for interaction } \\
\text { index }=0.71\end{array}$ & 167 , interaction \\
\hline
\end{tabular}

All tests were adjusted by age, sex and H. pylori infection. Statistically significant interaction was highlighted in bold (P values $<0.05$ ). Abbreviation: $\mathrm{GC}$, gastric cancer; $\mathrm{GA}$, atrophic gastritis; $\mathrm{CON}$, healthy controls.

Table 3: Epistatic effect of pair-wise interacting factors on the risks of gastric cancer and atrophic gastritis

\begin{tabular}{|c|c|c|c|c|c|c|}
\hline \multirow{2}{*}{ Interacted pair-wise SNPs } & \multirow{2}{*}{ Comparison } & \multirow{2}{*}{ Subset } & \multicolumn{2}{|c|}{ GA vs. CON } & \multicolumn{2}{|c|}{ GC vs. CON } \\
\hline & & & $\mathrm{OR}(95 \% \mathrm{CI})$ & $\mathrm{P}$ & $\mathrm{OR}(95 \% \mathrm{CI})$ & $\mathrm{P}$ \\
\hline \multirow{4}{*}{$\begin{array}{l}P G C \text { rs6912200 interacted with PTPN11 } \\
\text { rs } 12229892\end{array}$} & $P G C$ rs6912200 TT/TC vs. CC & PTPN11 rs12229892 GG & $0.65(0.43,0.99)$ & 0.043 & $0.56(0.35,0.90)$ & 0.016 \\
\hline & & PTPN11 rs12229892 AA/GA & $1.32(0.99,1.76)$ & 0.059 & $1.12(0.83,1.51)$ & 0.451 \\
\hline & PTPN11 rs12229892 AA/GA vs. GG & $P G C$ rs $6912200 \mathrm{CC}$ & $0.50(0.32,0.79)$ & 0.003 & $0.59(0.37,0.94)$ & 0.027 \\
\hline & & $P G C \mathrm{rs} 6912200 \mathrm{TT} / \mathrm{TC}$ & $1.00(0.77,1.29)$ & 0.982 & $1.11(0.84,1.46)$ & 0.466 \\
\hline \multirow{4}{*}{$\begin{array}{l}P G C \text { rs } 4711690 \text { interacted with } I L 1 B \\
\text { rs } 1143623\end{array}$} & $P G C$ rs $4711690 \mathrm{GG} / \mathrm{GC}$ vs $\mathrm{CC}$ & $I L 1 B$ rs $1143623 \mathrm{GG}$ & $0.81(0.59,1.10)$ & 0.176 & $1.07(0.77,1.50)$ & 0.683 \\
\hline & & $I L 1 B \mathrm{rs} 1143623 \mathrm{GC} / \mathrm{CC}$ & $0.76(0.60,0.96)$ & 0.021 & $0.69(0.5$ & 0.006 \\
\hline & ILIB rs $1143623 \mathrm{GC} / \mathrm{CC}$ vs GG & $P G C$ rs $4711690 \mathrm{CC}$ & $1.05(0.82,1.35)$ & 0.690 & $1.18(0.89,2.56)$ & 0.254 \\
\hline & & $P G C \mathrm{rs} 4711690 \mathrm{GG} / \mathrm{GC}$ & $0.99(0.73,1.34)$ & 0.946 & $0.76(0.55,1.06)$ & 0.102 \\
\hline \multirow{4}{*}{$\begin{array}{l}\text { PTPN11 rs12229892 interacted with } I L 1 B \\
\text { rs1143623 }\end{array}$} & PTPN11 rs12229892 AA/GA vs. GG & $I L 1 B$ rs $1143623 \mathrm{GG}$ & $0.76(0.55,1.07)$ & 0.114 & $0.74(0.52,1.07)$ & 0.110 \\
\hline & & $I L 1 B$ rs $1143623 \mathrm{GC} / \mathrm{CC}$ & $1.03(0.80,1.31)$ & 0.837 & $1.23(0.93,1.62)$ & 0.153 \\
\hline & $I L 1 B$ rs $1143623 \mathrm{GC} / \mathrm{CC}$ vs GG & PTPN11 rs12229892 GG & $0.84(0.60,1.17)$ & 0.295 & $0.68(0.47,1.00)$ & 0.050 \\
\hline & & PTPN11 rs12229892 AA/GA & $1.11(0.88,1.41)$ & 0.369 & $1.14(0.88,1.47)$ & 0.331 \\
\hline
\end{tabular}

All tests were adjusted by age, sex and H. pylori infection. Statistically significant associations were highlighted in bold (P values $<0.05$ ). Abbreviation: GC, gastric cancer; GA, atrophic gastritis; CON, healthy controls.

\section{Interactions involving multiple polymorphisms of PGC, PTPN11, and IL1B genes}

Next, we explored potential three- and four-way interactions among the four polymorphisms involved in significant pairwise interactions. A three-way interaction between $P G C$ rs 4711690 CG/GG, $P G C$ rs6912200 CT/ TT, and PTPN11 rs12229892 GA/AA was significantly associated with atrophic gastritis risk $(P$ value for interaction $=0.048$, interaction index $=2.82$ ) (Table 4). We analyzed the ORs by dividing the combined population into four subgroups based on the number of interacting genotypes (Table 5). A significant dosage effect was observed, with an increasing number of protective genotypes being associated with a decreasing risk of atrophic gastritis $\left(\mathrm{P}_{\text {trend }}=0.005\right)$. Four-way interactions among the four SNPs in relation to the risks of gastric cancer or atrophic gastritis did not reach statistical significance (Supplementary Table 5).
Effect modification of $H$. pylori infection on the interaction between $P G C$ rs4711690, $P G C$ rs6912200, and PTPN11 rs12229892

Intriguingly, all loci involved in the significant three-way interaction detailed above have also been shown to have interaction effects with $H$. pylori infection, as previously described $[7,17]$. We therefore felt that it was important to evaluate whether $H$. pylori infection modifies the effect of this three-way genetic interaction on the risk of atrophic gastritis. We first tested the effect modification of $H$. pylori on the interaction strength in a stratified analysis according to $H$. pylori infection status (Table 4). The interaction index was 0.96 in the $H$. pylori-negative subpopulation $(P=0.960)$, whereas it was 6.13 in the $H$. pylori-positive subpopulation $(P=0.029)$, suggesting that the interaction effect on atrophic gastritis risk is restricted to the cases infected with $H$. pylori.

We further tested the effect modification of $\mathrm{H}$. pylori on the cumulative effect of the three interacting SNPs. We used the Breslow-Day test to compare the differences between the ORs of each comparison in H. pylori-negative 
Table 4: Three-way interaction effect of $P G C$ rs4711690, $P G C$ rs6912200 and PTPN11 rs12229892 on atrophic gastritis risk

\begin{tabular}{|c|c|c|c|c|c|c|}
\hline \multirow{2}{*}{$\begin{array}{c}P G C \\
\text { rs } 4711690\end{array}$} & \multirow{2}{*}{$\begin{array}{c}P G C \\
\text { rs6912200 }\end{array}$} & \multirow{2}{*}{$\begin{array}{c}\text { PTPN11 } \\
\text { rs12229892 }\end{array}$} & \multirow{2}{*}{ CON (n) } & \multirow{2}{*}{ GA (n) } & \multicolumn{2}{|c|}{ GA vs CON } \\
\hline & & & & & $\mathrm{OR}(95 \% \mathrm{CI})$ & $\mathrm{P}$ \\
\hline \multicolumn{7}{|c|}{ Total population ${ }^{\mathrm{a}}$} \\
\hline $\mathrm{CC}$ & $\mathrm{CC}$ & GG & 22 & 26 & $1(\mathrm{ref})$ & \\
\hline $\mathrm{CC}$ & $\mathrm{CC}$ & GA/AA & 46 & 46 & $0.83(0.41,1.67)$ & 0.596 \\
\hline $\mathrm{CC}$ & $\mathrm{CT} / \mathrm{TT}$ & GG & 135 & 131 & $0.80(0.43,1.48)$ & 0.47 \\
\hline $\mathrm{CC}$ & $\mathrm{CT} / \mathrm{TT}$ & GA/AA & 271 & 251 & $0.77(0.42,1.39)$ & 0.38 \\
\hline $\mathrm{CG} / \mathrm{GG}$ & $\mathrm{CC}$ & GG & 33 & 45 & $1.15(0.56,2.38)$ & 0.707 \\
\hline $\mathrm{CG} / \mathrm{GG}$ & $\mathrm{CC}$ & GA/AA & 121 & 81 & $0.55(0.29,1.03)$ & 0.062 \\
\hline $\mathrm{CG} / \mathrm{GG}$ & $\mathrm{CT} / \mathrm{TT}$ & GG & 82 & 44 & $0.44(0.23,0.87)$ & 0.018 \\
\hline $\mathrm{CG} / \mathrm{GG}$ & $\mathrm{CT} / \mathrm{TT}$ & GA/AA & 169 & 140 & $0.69(0.38,1.28)$ & 0.238 \\
\hline
\end{tabular}

$P$ for interaction $=0.048$, interaction index $=2.82$

H. pylori-negative subpopulation ${ }^{\mathrm{b}}$

\begin{tabular}{ccccccc}
$\mathrm{CC}$ & $\mathrm{CC}$ & $\mathrm{GG}$ & 14 & 17 & $1(\mathrm{ref})$ & \\
$\mathrm{CC}$ & $\mathrm{CC}$ & $\mathrm{GA} / \mathrm{AA}$ & 29 & 13 & $0.35(0.13,0.91)$ & 0.032 \\
$\mathrm{CC}$ & $\mathrm{CT} / \mathrm{TT}$ & $\mathrm{GG}$ & 103 & 71 & $0.54(0.25,1.17)$ & 0.116 \\
$\mathrm{CC}$ & $\mathrm{CT} / \mathrm{TT}$ & $\mathrm{GA} / \mathrm{AA}$ & 199 & 106 & $0.41(0.20,0.88)$ & 0.021 \\
$\mathrm{CG} / \mathrm{GG}$ & $\mathrm{CC}$ & $\mathrm{GG}$ & 22 & 15 & $0.56(0.21,1.48)$ & 0.241 \\
$\mathrm{CG} / \mathrm{GG}$ & $\mathrm{CC}$ & $\mathrm{GA} / \mathrm{AA}$ & 88 & 27 & $0.24(0.10,0.54)$ & 0.001 \\
$\mathrm{CG} / \mathrm{GG}$ & $\mathrm{CT} / \mathrm{TT}$ & $\mathrm{GG}$ & 55 & 19 & $0.27(0.11,0.65)$ & 0.004 \\
$\mathrm{CG} / \mathrm{GG}$ & $\mathrm{CT} / \mathrm{TT}$ & $\mathrm{GA} / \mathrm{AA}$ & 121 & 37 & $0.24(0.11,0.54)$ & $<0.001$ \\
& & & & \multicolumn{4}{c}{$\mathrm{P}$ for interaction $=0.960$, interaction index $=0.96$}
\end{tabular}

H. pylori-positive subpopulation ${ }^{\mathrm{b}}$

$\begin{array}{ccccccc}\mathrm{CC} & \mathrm{CC} & \mathrm{GG} & 8 & 9 & 1(\mathrm{ref}) & \\ \mathrm{CC} & \mathrm{CC} & \mathrm{GA} / \mathrm{AA} & 17 & 33 & 1.83(0.59,5.66) & 0.294 \\ \mathrm{CC} & \mathrm{CT} / \mathrm{TT} & \mathrm{GG} & 32 & 60 & 1.71(0.60,4.91) & 0.32 \\ \mathrm{CC} & \mathrm{CT} / \mathrm{TT} & \mathrm{GA} / \mathrm{AA} & 72 & 145 & 1.92(0.70,5.24) & 0.203 \\ \mathrm{CG} / \mathrm{GG} & \mathrm{CC} & \mathrm{GG} & 27 & 25 & 2.50(0.76,8.19) & 0.131 \\ \mathrm{CG} / \mathrm{GG} & \mathrm{CC} & \mathrm{GA} / \mathrm{AA} & 48 & 103 & 1.51(0.53,4.35) & 0.444 \\ \mathrm{CG} / \mathrm{GG} & \mathrm{CT} / \mathrm{TT} & \mathrm{GG} & 17 & 9 & 0.86(0.29,2.61) & 0.794 \\ \mathrm{CG} / \mathrm{GG} & \mathrm{CT} / \mathrm{TT} & \mathrm{GA} / \mathrm{AA} & 28 & 17 & 2.05(0.74,5.70) & 0.17\end{array}$

$P$ for interaction $=0.029$, interaction index $=6.13$

$\overline{\mathrm{a}}$, these tests were adjusted by age, sex and H. pylori infection; ${ }^{\mathrm{b}}$, these tests were adjusted by age and sex. Statistically significant interactions were highlighted in bold ( $P$ values $<0.05$ ). Abbreviation: GA, atrophic gastritis; CON, healthy controls.

and -positive subgroups (Table 5). The subjects with one, two, or three variant genotypes had significantly different atrophic gastritis risks between the two subgroups based on $H$. pylori infection status ( $P$ value for Breslow-Day test $=0.049,0.020$, and 0.001 , respectively), suggesting that H. pylori infection can modify the cumulative effect of the three interacting SNPs.

\section{DISCUSSION}

Gastric cancer is presumed to be the cumulative result of interactions among many genes, with each gene only having a small effect. In this study, we found new SNP interactions among $H$. pylori-related host genes $P G C, P T P N 11$, and IL1B modifying the susceptibility to atrophic gastritis and gastric cancer. When we considered the host genetic effects alone, gene-gene interactions 
Table 5: Cumulative effect of the three interacting factors of $P G C$ rs6912200, $P G C$ rs4711690 and $P T P N 11$ rs12229892 on the risk of atrophic gastritis

\begin{tabular}{|c|c|c|c|c|c|c|c|c|c|c|}
\hline \multirow{2}{*}{ No. of interacting genotypes } & \multicolumn{3}{|l|}{ Total population } & \multicolumn{3}{|c|}{ H. pylori-negative subpopulation } & \multicolumn{3}{|c|}{ H. pylori-positive subpopulation } & \multirow{2}{*}{$\mathrm{Pbd}^{\mathrm{c}}$} \\
\hline & Controls/cases & $\mathrm{OR}(95 \% \mathrm{CI})$ & $\mathrm{P}^{\mathrm{a}}$ & Controls/cases & $\mathrm{OR}(95 \% \mathrm{CI})$ & $\mathrm{P}^{\mathrm{b}}$ & Controls/cases & $\mathrm{OR}(95 \% \mathrm{CI})$ & $\mathrm{P}^{\mathrm{b}}$ & \\
\hline 0 & $22 / 26$ & $1(\mathrm{ref})$ & & $14 / 17$ & & & $8 / 9$ & & & \\
\hline 1 & $214 / 222$ & $0.76(0.40,1.43)$ & 0.394 & $154 / 99$ & $0.51(0.24,1.08)$ & 0.078 & $60 / 126$ & $1.87(0.68,5.15)$ & 0.226 & 0.049 \\
\hline 2 & $474 / 376$ & $0.56(0.30,1.04)$ & 0.068 & $342 / 152$ & $0.35(0.17,0.72)$ & 0.005 & $132 / 224$ & $1.60(0.60,4.29)$ & 0.353 & 0.020 \\
\hline 3 & $169 / 140$ & $0.54(0.28,1.03)$ & 0.062 & $121 / 37$ & $0.24(0.11,0.54)$ & $4.96 \times 10^{-4}$ & $48 / 103$ & $2.02(0.73,5.63)$ & 0.179 & 0.001 \\
\hline
\end{tabular}

${ }^{a}$, these tests were adjusted by sex, age and H. pylori infection; ${ }^{b}$, these tests were adjusted by sex and age; ${ }^{\mathrm{c}}$, Breslow-Day test was employed to assess the homogeneity of stratum-specific ORs between $H$. pylori-negative and -positive subpopulations. Statistically significant results were highlighted in bold (P values $<0.05$ ). Abbreviation: $\mathrm{GC}$, gastric cancer; GA, atrophic gastritis; $\mathrm{CON}$, healthy controls.

consistently contributed to reduced risks of gastric cancer and/or atrophic gastritis, including two-way interactions: $P G C$ rs6912200-PTPN11 rs12229892, $P G C$ rs4711690$I L 1 B$ rs1143623 and PTPN11 rs12229892-IL1B rs1143623 and a three-way interaction: $P G C$ rs4711690- $P G C$ rs6912200-PTPN11 rs12229892. Interestingly, when the effect modification of $H$. pylori infection was evaluated, the cumulative effect of the three-way interaction of $P G C$ rs4711690- PGC rs6912200-PTPN11 rs12229892 was shown to differ by the status of $H$. pylori infection. The cumulative effects on atrophic gastritis susceptibility switched from being beneficial to being risky in the presence of $H$. pylori infection.

Since 2008, genome wide association studies (GWAS) have been performed to search for gastric cancer susceptibility loci [18], and several associated regions such as 1q22, 3q13.31, 5p13.1, 6p21.1, 8q24, 10q23, and $20 \mathrm{p} 13$ have been revealed[18-26]. However, finemapping susceptibility loci within these regions is still required. In this study, we selected four important host genes involving in the response to $H$. pylori infection. Among them, $P G C$ gene that plays an important role in gastric epithelial differentiation is located at $6 \mathrm{p} 21.1$. This region has been revealed to be an important susceptibility loci for multiple cancers such as noncardia gastric cancer, lung cancer, and esophageal squamous-cell carcinoma in a Chinese GWAS [25]. Currently, we focused on genegene interaction effect instead of individual gene effect, and $P G C$ was observed to have interaction effect with PTPN11 at $12 \mathrm{q} 24$ and $I L 1 B$ at $2 \mathrm{q} 14$ in susceptibility of gastric carcinogenesis. The interaction effect on atrophic gastritis risk between $P G C$ rs6912200 and PTPN11 rs12229892 $(\mathrm{OR}=0.60)$ was greater than the main effect of a single polymorphism, $P G C$ rs4711690 $(\mathrm{OR}=0.78)$ that was identified in our previous study [7]. Moreover, there was a three-way interaction, $P G C$ rs4711690- $P G C$ rs6912200-PTPN11 rs12229892, whose beneficial effect increased cumulatively with each additional SNP $(\mathrm{OR}=$ $0.73,0.56$, and 0.54 for one, two, and three interacting variant SNPs, respectively). The cumulative effect of the three SNPs was stronger than the effect of each SNP alone, which is indicative of a true interaction.

Notably, among the four significant interacting polymorphisms, only $P G C$ rs4711690 was previously found to have a main effect on disease risk while $P G C$ rs6912200, PTPN11 rs12229892, and IL1B rs1143623 had no such effect $[7,17]$. Indeed, such an interaction effect between polymorphisms of two or more genes in the absence of a significant main effect of any of them, is indicative of epistasis [27]. As such, the genetic effects of the PGC rs6912200, PTPN11 rs12229892, and IL1B rs1143623 polymorphisms on disease risks would have been missed had they not been tested jointly. Multiple studies have shown that epistatic gene-gene interactions confer susceptibility to various malignancies, such as breast cancer, lung cancer, and colorectal cancer [28-30]. In fact, only in rare cases does the disease appear to be monogenic and, generally, multiple genes are involved in tumor initiation and development. This addresses, in part, the apparent missing heritability of gastric cancer risk and provides novel insights into the multifactorial etiology of gastric cancer.

In the current study, the most significant epistatic gene-gene effect was between $P G C$ rs6912200 and PTPN11 rs12229892. Both TC/TT genotypes at rs6912200 and GA/AA genotypes at rs12229892 conferred a reduced risk of gastric cancer and atrophic gastritis in the absence of the other variant SNP, but showed no effect in its presence. In another epistatic interaction, the $I L 1 B$ rs1143623 GC/CC genotypes showed an association with a reduced gastric cancer risk only if the PTPN11 rs12229892 GA/AA genotypes were absent. These observations suggest that the effects of $P G C$ rs6912200, PTPN11 rs12229892, and IL1B rs1143623 on gastric cancer development principally rely on the status of the other SNP in each pair-wise interaction. However, the evidence for a direct functional relationship between the alleles of $P G C, P T P N 11$, and $I L 1 B$ is scarce. Nonetheless, it is possible to speculate that they interact with one another via various signal transduction pathways and our interactions may reflect this. For instance, the IL1B cytokine and Shp2 factor (PTPN11) could mutually activate each other through their related ERK and MAPK pathways [9-11, 31]. Activation of the ERK pathway could then promote the expression of the PGC protein [32]. Additionally, Shp2 has a central role in several other pathways coordinating various cellular processes in response to extracellular stimuli, including those affecting 
cell growth and motility [33]. Genetic variants of any gene within these networks could potentially have an effect on the action of the other genes and could thus disturb the balance of homeostasis of gastric epithelial cells. Since we only included tagSNPs of the genes of interest in this study, other functional SNPs covered by the tagSNPs but not involved in this study yet could also participate in the interaction. Further independent study that covered more SNPs in addition to tagSNPs are warranted, and comprehensive function experiment involved two or more genes would be informative to estimate the role of susceptibility loci that directly affect gastric cancer development.

The phenomenon of an effect modification by H. pylori infection observed in the PTPN11 and PGC interaction may provide an important hint to help prevent gastric cancer by eradicating $H$. pylori in susceptible people. Of importance, PTPN11 and PGC function as critical host genes in the network of $H$. pylori pathogenicity in the gastric epithelium [8-14]. One of the most important virulence factors of $H$. pylori, CagA, can activate the PTPN11 encoded protein, Shp2, and its related MAPK pathways and induce epithelial transformation, proliferation, and inflammation[34]. PGC protein acts as a critical gastric effector of signals stimulated by the LPS of CagA (+) H. pylori [35]. The effect modification of $H$. pylori on such host genes might be ubiquitous in the stomach but has been ignored in many studies. We previously found that each of $P G C$ rs4711690, $P G C$ rs6912200, and PTPN11 rs12229892 had an interaction effect with $H$. pylori [7]. In the current study, significant effect heterogeneity by $H$. pylori infection status was also observed for the three-way interaction of $P G C$ rs 4711690 , $P G C$ rs6912200, and PTPN11 rs12229892. Moreover, the interaction strength was found to be enhanced in the $H$. pylori-infected subpopulation. This phenomenon indicated an effect modification by $H$. pylori on the cumulative effect of interacting host factors. It is plausible that $H$. pylori may function as a bridge for SNP-SNP interactions between the PTPN11 and PGC genes, in which certain virulence factors or modulins of this microbe may modify the host gene's innate function. Despite previous efforts, the mechanism by which $H$. pylori interacts with each polymorphism remains elusive. Further functional research concerning the role of $H$. pylori in $P G C$ and PTPN11 interactions is warranted, which may, in part, compensate for the probability of false positive/negative findings.

Due to a retrospective study design, the data of enrolled subjects' basic characteristic were also retrospectively extracted from registered databank. However, some information of a portion of the enrolled subjects was lacked, such as smoking and drinking status, family history, and economic status. Accordingly, when we measured the association strength, only the status of sex, age and $H$. pylori infection were adjusted, which may be a main limitation of the current study. Therefore, more potential confounding factors should be included in further independent replication study.

In summary, we observed novel SNP interactions among $P G C, P T P N 11$, and $I L 1 B$ which modified the risks of gastric cancer and atrophic gastritis and we provided important hints of effect modification by $H$. pylori infection on the cumulative effect of $P G C$ rs6912200, $P G C$ rs 4711690 , and PTPN11 rs12229892. Better understanding the gene-gene and gene-environment interactions could provide important insights into the etiology of gastric cancer. The potential impact of $H$. pylori infection on genetic susceptibility in the prediction and prevention of gastric cancer needs to be considered in future studies.

\section{MATERIALS AND METHODS}

\section{Study population}

This study was approved by the Human Ethics Review Committee of First Affiliated Hospital of China Medical University. Written informed consent was obtained from each participant. A total of 2897 subjects consisting of 1276 healthy controls, 907 cases of atrophic gastritis, and 714 cases of gastric cancer were included in the current study. A full description of the inclusion criteria, diagnostic criteria, and characteristics of the study population has been reported previously [7, 17]. Briefly, all the subjects were Chinese and living in northern China. They were recruited between 2002 and 2011 from a health check program for gastric cancer screening or from hospitals in Zhuanghe or Shenyang in Liaoning Province, China. The diagnoses of all the study subjects were independently made by two gastrointestinal pathologists according to the Consensus on Chronic Gastritis formulated at the National Symposium in combination with the updated Sydney System and the World Health Organization (WHO) criteria[36-38]. The healthy control subjects in the current study comprised individuals with normal stomachs or with only slight superficial gastritis without atrophic lesions or intestinal metaplasia. Subjects who had a history of other malignant tumors were excluded.

\section{SNP selection and genotyping}

As described in our previous studies [7, 17], we employed a two-step approach to select tagSNPs for the genes of interest. Briefly, Haploview software (http:// www.broadinstitute.org/mpg/haploview) was used to minimize the number of SNPs that needs to be genotyped and FastSNP search (http://FastSNP.ibms.sinica.edu.tw/) was performed to predict their functional effects. The 
predicted function of each tagSNP selected in this study were summarized in Supplementary table 1. Genomic DNA was isolated from peripheral blood lymphocytes by the routine phenol-chloroform method. The genotypes of 13 tagSNPs (rs4711690, rs6458238, rs9471643, rs6941539, rs6912200, rs3789210, and rs6939861 of PGC; rs10983755 and rs11536878 of TLR4; rs 12229892 of PTPN11; and rs1143623, rs1143627, and rs1143643 of $I L 1 B$ ) were selected and assessed by the Sequenom MassARRAY platform (Sequenom, San Diego, CA, USA) according to the manufacturer's instructions [7, 17]. Each DNA sample was diluted to a working concentration of $50 \mathrm{ng} / \mu \mathrm{L}$ for genotyping. All samples were randomly placed on the 384-well plates and the operator confirming the SNP genotyping calls was blinded to disease status. Randomly selected samples had repeat genotypes performed and $100 \%$ concordance was confirmed.

\section{ELISA assessment of $\mathrm{H}$. pylori immunoglobulin G (IgG) in serum}

Serum H. pylori IgG levels were determined by enzyme-linked immunosorbent assay $(H$. pylori $\mathrm{IgG}$ ELISA kit, BIOHIT, Helsinki, Finland). A reading > 34 enzyme immune units was defined to be $H$. pylori seropositive.

\section{Statistical analysis}

We set the combination of common genotypes as the reference and employed the likelihood-ratio test to assess the SNP-SNP interaction effects by comparing the model that only contained the main effects of each factor with the full model that also contained the interaction terms. Odds ratios (ORs) with their 95\% confidence intervals (CI) were calculated as measures of associations adjusted by sex, age and $H$. pylori infection unless the $H$. pylori has been used as a stratified factor. The Cochrane-Armitage test for linear trend was used to examine whether there was a dosage effect on disease risk with an increasing number of interacting SNP genotypes. To compare the effect modification of $H$. pylori status on the cumulative effect of the interacting SNPs on disease risk, the Breslow-Day test was employed to assess the homogeneity of stratumspecific ORs across different subgroups. All the analyses were performed using SPSS 16.0 software (SPSS Inc., Chicago, IL, USA) and Stata version 11.0 (StataCorp., College Station, TX, USA). All $P$ values were two sided, and $P$ values $<0.05$ were considered statistically significant.

\section{ACKNOWLEDGMENTS}

This study is supported by grants from National Basic Research Program of China (973 Program Ref
No.2010CB529304) and the National Natural Science Foundation of China (Ref No. 31200968)

\section{CONFLICTS OF INTEREST}

None declared.

\section{Authors' contributions}

Yuan Yuan conceived and designed this study and revised the manuscript. Caiyun He was responsible for the experiment and performed data interpretation and wrote the paper. Huakang Tu was responsible for the statistical analyses partly. Liping Sun and Qian Xu performed data interpretation partly. Jing Jingjing and Nannan Dong preformed the experiment partly. All authors read and approved the final manuscript, and do not have a commercial or other association that might pose a conflict of interest.

\section{FUNDING}

This study is supported by grants from National Basic Research Program of China (973 Program Ref No.2010CB529304) and the National Natural Science Foundation of China (Ref No. 31200968).

\section{Abbreviations}

PGC, pepsinogen C; PTPN11, protein tyrosine phosphatase, non-receptor type 11; TLR4, Toll-like receptor 4; IL1B, interleukin-1B; H. pylori, Helicobacter pylori; LPS, lipopolysaccharide; CagA, cytotoxinassociated antigen $\mathrm{A}$; $\mathrm{NF}-\mathrm{Kb}$, nuclear factor-kappa B; MAPK, mitogen-activated protein kinase; SNP, single nucleotide polymorphism; WHO, World Health Organization; IgG, immunoglobulin G; OR, Odds ratio; CI, confidence interval.

\section{REFERENCES}

1. Roukos DH. Assessing both genetic variation (SNPs/CNVs) and gene-environment interactions may lead to personalized gastric cancer prevention. Expert Rev Mol Diagn 2009; 9: 1-6.

2. Hancock DB, Scott WK. Population-based case-control association studies Curr Protoc Hum Genet 2012; Chapter 1: Unit1 17.

3. Moore JH. The ubiquitous nature of epistasis in determining susceptibility to common human diseases. Hum Hered 2003; 56: 73-82.

4. Fang G, Haznadar M, Wang W, Yu H, Steinbach M, Church TR, Oetting WS, Van Ness B, Kumar V. High-order SNP combinations associated with complex diseases: efficient 
discovery, statistical power and functional interactions. PLoS One 2012; 7: e33531.

5. Fock KM, Katelaris P, Sugano K, Ang TL, Hunt R, Talley NJ, Lam SK, Xiao SD, Tan HJ, Wu CY, Jung HC, Hoang $\mathrm{BH}$, Kachintorn U, et al. Second Asia-Pacific Consensus Guidelines for Helicobacter pylori infection. J Gastroenterol Hepatol 2009; 24: 1587-1600.

6. He C, Chen M, Liu J, Yuan Y. Host genetic factors respond to pathogenic step-specific virulence factors of Helicobacter pylori in gastric carcinogenesis. Mutat Res 2014; 759C: 1426.

7. He C, Tu H, Sun L, Xu Q, Li P, Gong Y, Dong N, Yuan $\mathrm{Y}$. Helicobacter pylori-related host gene polymorphisms associated with susceptibility of gastric carcinogenesis: a two-stage case-control study in Chinese. Carcinogenesis 2013; 34: 1450-1457.

8. Katoh M. Dysregulation of stem cell signaling network due to germline mutation, SNP, Helicobacter pylori infection, epigenetic change and genetic alteration in gastric cancer. Cancer Biol Ther 2007; 6: 832-839.

9. Shah S, King EM, Chandrasekhar A, Newton R. Roles for the Mitogen-activated Protein Kinase (MAPK) Phosphatase, DUSP1, in Feedback Control of Inflammatory Gene Expression and Repression by Dexamethasone. J Biol Chem 2014; 289: 13667-13679.

10. Glossop JR, Cartmell SH. Effect of fluid flow-induced shear stress on human mesenchymal stem cells: differential gene expression of IL1B and MAP3K8 in MAPK signaling. Gene Expr Patterns 2009; 9: 381-388.

11. Arana-Argaez VE, Delgado-Rizo V, Pizano-Martinez OE, Martinez-Garcia EA, Martin-Marquez BT, Munoz-Gomez A, Petri MH, Armendariz-Borunda J, Espinosa-Ramirez G, Zuniga-Tamayo DA, Herrera-Esparza R, Vazquez-Del Mercado M. Inhibitors of MAPK pathway ERK1/2 or p38 prevent the IL-1 \{beta\}-induced up-regulation of SRP72 autoantigen in Jurkat cells. J Biol Chem 2010; 285: 3282432833.

12. Di Mario F, Moussa AM, Cavallaro LG, Caruana P, Merli R, Bertolini S, Iori V, Cavestro GM, Dal Bo N, Pilotto A, Franze A, Leandro G. Clinical usefulness of serum pepsinogen II in the management of Helicobacter pylori infection. Digestion 2004; 70: 167-172.

13. Massarrat S, Haj-Sheykholeslami A, Mohamadkhani A, Zendehdel N, Aliasgari A, Rakhshani N, Stolte M, Shahidi SM. Pepsinogen II Can Be a Potential Surrogate Marker of Morphological Changes in Corpus before and after H. pylori Eradication. Biomed Res Int 2014; 2014: 481607.

14. Yasugi S. Epithelial cell differentiation during stomach development. Hum Cell 2000; 13: 177-184.

15. Hassan MI, Toor A, Ahmad F. Progastriscin: structure, function, and its role in tumor progression. J Mol Cell Biol 2010; 2: 118-127.

16. El-Omar EM, Ng MT, Hold GL. Polymorphisms in Tolllike receptor genes and risk of cancer. Oncogene 2008; 27 :
244-252.

17. He CY, Sun LP, Xu Q, Liu JW, Jiang JY, Dong NN, Yuan Y. PGC TagSNP and Its Interaction with H. pylori and Relation with Gene Expression in Susceptibility to Gastric Carcinogenesis. PLoS One 2014; 9: e115955.

18. Sakamoto H, Yoshimura K, Saeki N, Katai H, Shimoda T, Matsuno Y, Saito D, Sugimura H, Tanioka F, Kato S, Matsukura N, Matsuda N, Nakamura T, et al. Genetic variation in PSCA is associated with susceptibility to diffuse-type gastric cancer. Nat Genet 2008; 40: 730-740.

19. Zhang H, Jin G, Li H, Ren C, Ding Y, Zhang Q, Deng B, Wang J, Hu Z, Xu Y, Shen H. Genetic variants at 1q22 and 10q23 reproducibly associated with gastric cancer susceptibility in a Chinese population. Carcinogenesis 2011; 32: 848-852.

20. Zeng Z, Wu X, Chen F, Yu J, Xue L, Hao Y, Wang Y, Chen M, Sung JJ, Hu P. Polymorphisms in prostate stem cell antigen gene rs2294008 increase gastric cancer risk in Chinese. Mol Carcinog 2011; 50: 353-358.

21. Yang XX, Li FX, Zhou CP, Hu NY, Wu YS, Li M. Association of genetic polymorphisms at 1q22 but not 10q23 with gastric cancer in a southern Chinese population. Asian Pac J Cancer Prev 2012; 13: 2519-2522.

22. Wang M, Zhang R, He J, Qiu L, Li J, Wang Y, Sun M, Yang Y, Wang J, Yang J, Qian J, Jin L, Ma H, et al. Potentially functional variants of PLCE1 identified by GWASs contribute to gastric adenocarcinoma susceptibility in an eastern Chinese population. PLoS One 2012; 7: e31932.

23. Shi Y, Hu Z, Wu C, Dai J, Li H, Dong J, Wang M, Miao X, Zhou Y, Lu F, Zhang H, Hu L, Jiang Y, et al. A genomewide association study identifies new susceptibility loci for non-cardia gastric cancer at 3q13.31 and 5p13.1. Nat Genet 2011; 43: 1215-1218.

24. Saeki N, Ono H, Sakamoto H, Yoshida T. Genetic factors related to gastric cancer susceptibility identified using a genome-wide association study. Cancer Sci. 2013; 104:1-8.

25. Jin G, Ma H, Wu C, Dai J, Zhang R, Shi Y, Lu J, Miao X, Wang M, Zhou Y, Chen J, Li H, Pan S, et al. Genetic variants at 6 p21.1 and 7 p15.3 are associated with risk of multiple cancers in han chinese. Am J Hum Genet 2012; 91: 928-934.

26. Abnet CC, Freedman ND, Hu N, Wang Z, Yu K, Shu XO, Yuan JM, Zheng W, Dawsey SM, Dong LM, Lee MP, Ding T, Qiao YL, et al. A shared susceptibility locus in PLCE1 at 10q23 for gastric adenocarcinoma and esophageal squamous cell carcinoma. Nat Genet 2010; 42: 764-767.

27. Carlborg O, Haley CS. Epistasis: too often neglected in complex trait studies? Nat Rev Genet 2004; 5: 618-625.

28. Ritchie MD, Hahn LW, Roodi N, Bailey LR, Dupont WD, Parl FF, Moore JH. Multifactor-dimensionality reduction reveals high-order interactions among estrogen-metabolism genes in sporadic breast cancer. Am J Hum Genet 2001; 69: 138-147.

29. Chu M, Zhang R, Zhao Y, Wu C, Guo H, Zhou B, Lu J, Shi 
Y, Dai J, Jin G, Ma H, Dong J, Wei Y, et al. A genomewide gene-gene interaction analysis identifies an epistatic gene pair for lung cancer susceptibility in Han Chinese. Carcinogenesis 2014; 35: 572-577.

30. Jiao S, Hsu L, Berndt S, Bezieau S, Brenner H, Buchanan D, Caan BJ, Campbell PT, Carlson CS, Casey G, Chan AT, Chang-Claude J, Chanock S, et al. Genome-wide search for gene-gene interactions in colorectal cancer. PLoS One 2012; 7: e52535.

31. Hatakeyama M. Oncogenic mechanisms of the Helicobacter pylori CagA protein. Nat Rev Cancer 2004; 4: 688-694.

32. Fiorucci S, Distrutti E, Federici B, Palazzetti B, Baldoni M, Morelli A, Cirino G. PAR-2 modulates pepsinogen secretion from gastric-isolated chief cells. Am J Physiol Gastrointest Liver Physiol 2003; 285: G611-620.

33. Neel BG, Gu H, Pao L. The 'Shp'ing news: SH2 domaincontaining tyrosine phosphatases in cell signaling. Trends Biochem Sci 2003; 28: 284-293.

34. Suzuki M, Mimuro H, Kiga K, Fukumatsu M, Ishijima N, Morikawa H, Nagai S, Koyasu S, Gilman RH, Kersulyte D, Berg DE, Sasakawa C. Helicobacter pylori CagA phosphorylation-independent function in epithelial proliferation and inflammation. Cell Host Microbe 2009; 5: 23-34.

35. Young GO, Brown S, Stemmet N, Lastovica AJ, Marks IN, Modlin IM, Kidd M. The pepsinogen releasing effect of Helicobacter pylori lipopolysaccharide. Helicobacter 2002; 7: 30-38.

36. Hamilton SR, Aaltonen LA. World Health Organization classification of tumours. Pathology and genetics of tumors of the digestive system. Lyon:IARC Press 2000: 237-240.

37. Dixon MF, Genta RM, Yardley JH, Correa P. Classification and grading of gastritis. The updated Sydney System. International Workshop on the Histopathology of Gastritis, Houston 1994. Am J Surg Pathol 1996; 20: 1161-1181.

38. Lin SB, Yu ZL, Hu PJ, Wang CW, Xu GM, Xiao SD, Shi R, Liu WZ. Consensus on chronic gastritis formulated at the national symposium. Zhonghua Xiaohua Zazhi 2000; 20: 199-201. 